PROCEEDINGS OF THE

AMERICAN MATHEMATICAL SOCIETY

Volume 133, Number 8, Pages 2283-2295

S 0002-9939(05)07772-5

Article electronically published on March 14, 2005

\title{
INVARIANT MEASURES FOR GENERAL(IZED) INDUCED TRANSFORMATIONS
}

\author{
ROLAND ZWEIMÜLLER
}

(Communicated by Michael Handel)

\begin{abstract}
We show that the general(ized) induced transformation $T^{\tau}$ derived from an ergodic measure preserving transformation $T$ by means of an inducing time $\tau$ has an invariant measure canonically related to that of the original system iff a suitable induced version of $\tau$ is integrable. Moreover, we prove an Abramov-type entropy formula.
\end{abstract}

\section{INTRODUCTION}

Let $T$ be a nonsingular transformation on the $\sigma$-finite measure space $(X, \mathcal{A}, m)$, i.e. $T: X \rightarrow X$ is a measurable map satisfying $m \circ T^{-1} \ll m$. The first return time $\varphi_{Y}(x):=\min \left\{n \geq 1: T^{n} x \in Y\right\}, x \in Y$, of a set $Y \in \mathcal{A}^{+}(m):=\{E \in \mathcal{A}: m(E)>$ $0\}$ is a measurable function $\varphi_{Y}: Y \rightarrow \overline{\mathbb{N}}:=\mathbb{N} \cup\{\infty\}$. If $\varphi_{Y}<\infty$ a.e. on $Y$, i.e. if $Y$ is a recurrent set, it gives rise to the first return (or induced) map $T_{Y}: Y \rightarrow Y$ defined a.e. by $T_{Y} x:=T^{\varphi_{Y}(x)} x$, which is a very useful classical construction in ergodic theory; cf. [Ka] and [He]. Most important,

$$
\text { if } \mu \ll m \text { is } T \text {-invariant, then } \nu:=\left.\mu\right|_{\mathcal{A} \cap Y} \text { is } T_{Y} \text {-invariant, }
$$

$$
\text { and }
$$

if $\nu \ll m$ is $T_{Y}$-invariant, then $\nu=\left.\mu\right|_{\mathcal{A} \cap Y}$ for the $T$-invariant measure $\mu \ll m$ given by $\mu(E):=\sum_{n \geq 0} \nu\left(\left\{\varphi_{Y}>n\right\} \cap T^{-n} E\right)$.

Turning to a frequently-used generalization of this concept, we shall call a measurable function $\tau: Y \rightarrow \overline{\mathbb{N}}$ an inducing time $(\bmod m)$ for $T$ on $Y \in \mathcal{A}^{+}(m)$ if it is finite a.e. and $T^{\tau} x:=T^{\tau(x)} x \in Y$ for a.e. $x \in Y$. $T^{\tau}$ is then a nonsingular transformation on $\left(Y, \mathcal{A} \cap Y,\left.m\right|_{\mathcal{A} \cap Y}\right)$. We call $T^{\tau}$ the transformation that $T$ and $\tau$ induce on $Y$. Given such a $\tau$ and any measure $\nu$ on $(Y, \mathcal{A} \cap Y$ ) (which we will tacitly extend to $(X, \mathcal{A})$ by letting $\left.\nu\left(Y^{c}\right):=0\right)$, we define a new measure $\tau \times_{T} \nu$ on $(X, \mathcal{A})$ by

$$
\left(\tau \times_{T} \nu\right)(E):=\sum_{n \geq 0} \nu\left(\{\tau>n\} \cap T^{-n} E\right), \quad E \in \mathcal{A} .
$$

Received by the editors July 23, 2003.

2000 Mathematics Subject Classification. Primary 28D05, 28D20, 37A05, 60G10, 60G40.

This research was partially supported by the Austrian Science Foundation FWF, project P14734-MAT, and by an APART [Austrian programme for advanced research and technology] fellowship of the Austrian Academy of Sciences.

(C)2005 American Mathematical Society Reverts to public domain 28 years from publication 
Equivalently, $\int f d\left(\tau \times_{T} \nu\right)=\int \sum_{n \geq 0} 1_{\{\tau>n\}}\left(f \circ T^{n}\right) d \nu$ for measurable $f \geq 0$ on $(X, \mathcal{A})$. In the special case of first return times $\tau=\varphi_{Y}$, we always have

$$
\left.\left(\varphi_{Y} \times_{T} \nu\right)\right|_{\mathcal{A} \cap Y}=\left.\nu\right|_{\mathcal{A} \cap Y} \quad \text { for any } \nu \text { on }(Y, \mathcal{A} \cap Y)
$$

since $\left\{\varphi_{Y}>n\right\} \cap T^{-n} Y=\emptyset$ for $n \geq 1$. Notice also that $\tau \times_{T} \nu$ is linear in $\nu$, and that for $T$-invariant measures $\nu$ and constant $\tau \equiv t \in \mathbb{N}$, we have $\tau \times_{T} \nu=t \cdot \nu$. The importance of this construction is due to the following fact, the "if"-part of which is well known.

Proposition 1.1 (Invariance and absolute continuity of $\left.\tau \times_{T} \nu\right)$. Let $T$ be a nonsingular transformation on the $\sigma$-finite measure space $(X, \mathcal{A}, m)$, and let $\tau$ be an inducing time $(\bmod m)$ for $T$ on $Y \in \mathcal{A}^{+}(m)$. Let $\nu$ be a measure on $(Y, \mathcal{A} \cap Y)$. Then

$$
\tau \times_{T} \nu \ll m \quad \text { iff } \quad \nu \ll m,
$$

and

$$
\tau \times_{T} \nu \text { is T-invariant iff } \quad \nu \text { is } T^{\tau} \text {-invariant. }
$$

Moreover,

$$
\left(\tau \times_{T} \nu\right)(X)=\sum_{n \geq 0} \nu(\{\tau>n\})=\int_{Y} \tau d \nu .
$$

Notice that we do not claim that $\tau \times_{T} \nu$ has to be $\sigma$-finite, even if $\nu$ is finite. In fact, $\tau \times_{T} \nu$ may have density $\infty$ w.r.t. $m$; see Lemma 2.1 and Example 2.2 below.

Proof. We briefly recall the argument; compare $[\mathrm{Th}]$. Since for any $E \in \mathcal{A}$,

$$
\left(\tau \times_{T} \nu\right)\left(T^{-1} E\right)=\sum_{n \geq 1} \nu\left(\{\tau>n\} \cap T^{-n} E\right)+\sum_{n \geq 0} \nu\left(\{\tau=n\} \cap T^{-n} E\right),
$$

we have $\left(\tau \times_{T} \nu\right)\left(T^{-1} E\right)=\left(\tau \times_{T} \nu\right)(E)$ iff

$$
\nu(Y \cap E)=\nu(E)=\sum_{n \geq 0} \nu\left(\{\tau=n\} \cap T^{-n} E\right)=\nu\left(\left(T^{\tau}\right)^{-1}(Y \cap E)\right),
$$

implying the first statement. The assertion about absolute continuity follows from $\tau \times_{T} \nu \geq \nu$ and nonsingularity of $T$.

Proposition 1.1 is the standard tool for constructing an invariant measure $\mu$ for $T$ from an invariant measure $\nu$ for $T^{\tau}$ : Given a transformation $T$ which we wish to investigate, we may be able to find $Y \in \mathcal{A}^{+}(m)$ and an inducing time $\tau$ on $Y$ such that $T^{\tau}$ is a more convenient map than $T$, preserving some measure $\nu=\nu \circ\left(T^{\tau}\right)^{-1} \ll m$. The proposition then provides us with an explicit formula for an invariant measure $\mu:=\tau \times_{T} \nu \ll m$ for $T$, which under natural additional assumptions inherits properties like ergodicity and conservativity from $T^{\tau} ; \mathrm{cf}$. [Th] (therefore $\mu=\tau \times_{T} \nu$ is the proper condition to ensure that the two measurepreserving systems are intimately related). Examples of applications of this scheme are abundant in the literature.

Notice, however, that the proposition does not enable us to go the other way, it merely ensures that, given $\mu$ and $\tau$, a measure $\nu$ solving $\mu=\tau \times_{T} \nu$ would be $T^{\tau}$-invariant, but it does not provide any information about the existence of such 
a measure. It is this converse which most of the present note is devoted to:

Question: Let $T$ be a measure preserving transformation (m.p.t.) on the $\sigma$-finite measure space $(X, \mathcal{A}, \mu)$, and let $\tau$ be an inducing time for $T$ on $Y$. Does $T^{\tau}$ preserve some measure $\nu \ll \mu$ with $\mu=\tau \times_{T} \nu ?$

Although we are going to view this question as one of abstract ergodic theory, we emphasize that the answer turns out to be a useful tool in situations where the analysis of specific dynamical systems requires the use of some general induced transformation not a priori known to possess a suitable invariant measure. These applications being rather technical, we are not going to discuss them in detail here, but refer to [PS] for an interesting example.

In view of Proposition 1.1 our question is equivalent to asking whether there is any measure $\nu$ on $(Y, \mathcal{A} \cap Y)$ solving the equation $\mu=\tau \times_{T} \nu$. In the case of

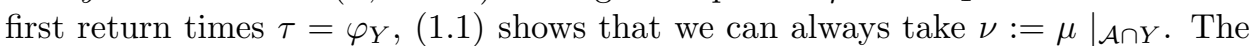
only other general result I am aware of applies to the first passage time of a set $Y \in \mathcal{A}^{+}(\mu), \tau(x)=\varphi^{Y}(x):=\min \left\{n \geq 0: T^{n} x \in Y\right\}+1, x \in X$, for which $T^{\tau}$ (the first passage map or jump transformation of $Y$ ) is weakly isomorphic to $T_{Y}$; cf. chapter 19 of $\mathrm{Sc}$.

We are going to provide an answer for arbitrary inducing times. Let us state the results for finite $\mu$ (see Section 4 for comments on the infinite measure case). The key step will be to show (in Section 3) that $\mu$-integrability of $\tau$ is a sufficient condition:

Theorem 1.1 (Invariant measure for $T^{\tau}$ if $\tau$ is $\mu$-integrable). Let $T$ be an ergodic measure-preserving transformation on the finite measure space $(X, \mathcal{A}, \mu)$, and let $\tau$ be an inducing time $(\bmod \mu)$ for $T$ on $Y \in \mathcal{A}^{+}(\mu)$. If $\int_{Y} \tau d \mu<\infty$, then $T^{\tau}: Y \rightarrow Y$ has an invariant measure $\nu \ll \mu$ satisfying $\mu=\tau \times_{T} \nu$.

Although it turns out that $\mu$-integrability is not a necessary condition (cf. Example 2.3 below), we will see that to avoid the kind of difficulties encountered there, we need only induce once more. We are going to prove the following characterization:

Theorem 1.2 (Invariant measure for $T^{\tau}$ iff $\tau$ has a $\mu$-integrable induced version). Let $T$ be an ergodic m.p.t. on the finite measure space $(X, \mathcal{A}, \mu)$, and let $\tau$ be an inducing time $(\bmod \mu)$ for $T$ on $Y \in \mathcal{A}^{+}(\mu)$. Then

$$
\mu=\tau \times_{T} \nu \quad \text { has a solution } \nu
$$

iff

there is some $Z \in \mathcal{A}^{+}(\mu), Z \subseteq Y$, such that $\int_{Z} \theta(\tau, Z) d \mu<\infty$, where $\varphi_{Z}^{\tau}(x):=\min \left\{n \geq 1:\left(T^{\tau}\right)^{n} x \in Z\right\}$, and $\theta(\tau, Z):=\sum_{k=0}^{\varphi_{Z}^{\tau}-1} \tau \circ\left(T^{\tau}\right)^{k}$.

Let us also point out that, formulated in probabilistic terms, our results can be interpreted as stationary sampling theorems:

Remark 1.1 (Stationary sampling). Let $\eta=\left(\eta_{n}\right)_{n \geq 0}$ be an ergodic stationary sequence on the probability space $(\Omega, \mathcal{F}, P)$ taking values in $\left(\Omega^{\prime}, \mathcal{F}^{\prime}\right)$, and $R: \Omega \rightarrow \mathbb{N}$ a random time measurable w.r.t. the $\sigma$-field generated by $\eta$ (but not necessarily a stopping time). Consider the the canonical shift-space representation of $\eta$, i.e. let $(X, \mathcal{A}, \mu):=\left(\bigotimes_{n \geq 0} \Omega^{\prime}, \bigotimes_{n \geq 0} \mathcal{F}^{\prime}, P \circ \Theta^{-1}\right)$, where $\Theta(\omega):=\left(\eta_{n}(\omega)\right)_{n \geq 0}$, so that $\eta_{n}=\pi\left(T^{n} \Theta(\omega)\right)$ with $\pi$ denoting the projection from $\bigotimes_{n \geq 0} \Omega^{\prime}$ onto its first factor, and $T$ is the shift on this product space, $\left(T \omega^{\prime}\right)_{n}=\omega_{n+1}^{\prime}$. Then $R=\tau \circ \Theta$ for 
some inducing time $\tau$ for $T$ on $X$, which gives a natural way to iterate $R$ by letting $R_{n}(\omega):=\tau\left(\left(T^{\tau}\right)^{n} \Theta(\omega)\right), n \geq 0$.

In general, the induced process $\eta^{R}=\left(\eta_{n}^{R}\right)_{n \geq 0}:=\left(\eta_{R_{n}}\right)_{n \geq 0}$ on $(\Omega, \mathcal{F}, P)$ is not stationary. However, Theorem 1.1 ensures that $\eta^{R}$ has a stationary distribution $Q$ absolutely continuous w.r.t. $P$ (and, for example, therefore satisfies a pointwise ergodic theorem w.r.t. $P$ ) as soon as $R$ has finite expectation. Theorem 1.2 completely characterizes those random times $R$ for which the same conclusion holds.

In the final section, we study the relation between the entropies of an m.p.t. $T$ and its induced transformations $T^{\tau}$, providing a generalized Abramov-type formula.

\section{Some PREPARATIONS AND EXAMPLES}

As an easy warm-up we observe the following.

Remark 2.1 (Uniqueness of solutions). Let $T$ be a conservative and ergodic (c.e.) measure-preserving transformation on the $\sigma$-finite measure space $(X, \mathcal{A}, \mu), Y \in$ $\mathcal{A}^{+}(\mu)$, and let $\tau$ be an inducing time for $T$ on $Y$. According to Proposition 1.1 any measure $\nu$ solving $\mu=\tau \times_{T} \nu$ is $\sigma$-finite and $T^{\tau}$-invariant, which determines $\nu$ up to a constant factor provided that $T^{\tau}$ is conservative ergodic on $\left(Y, \mathcal{A} \cap Y,\left.\mu\right|_{Y}\right)$; cf. Theorem 1.5.6 of [A0]. In general this need not be true, but it is in the most important case of first return maps, where the fact that $\left.\left(\varphi_{Y} \times_{T} \nu\right)\right|_{Y}=\nu$ also determines the normalization. Therefore, whenever $\nu$ is a measure on $(Y, \mathcal{A} \cap Y)$, we have

$$
\mu=\varphi_{Y} \times_{T} \nu \quad \text { iff } \quad \nu=\left.\mu\right|_{Y} .
$$

Easy counterexamples for the general case (with nonergodic $T^{\tau}$ ) can be obtained as follows: Take any partition $X=X_{0} \cup X_{1}$ with $\mu\left(X_{i}\right)>0$, and define $\tau: X \rightarrow \mathbb{N}$ by requiring that $\left.\tau\right|_{X_{i}}=\varphi_{X_{i}}$. Then it is straightforward to check that each $\nu_{i}:=$ $\left.\mu\right|_{\mathcal{A} \cap X_{i}}$ satisfies $\tau \times_{T} \nu_{i}=\varphi_{X_{i}} \times_{T} \nu_{i}=\mu$ (and so do all convex combinations).

Even if $T^{\tau}$ has an absolutely continuous invariant measure (a.c.i.m.) $\nu$, its action may take place on a different time scale than that of $T$ :

Lemma 2.1 (Dichotomy rule for $\tau \times_{T} \nu$ ). Let $T$ be an ergodic measure-preserving transformation on the finite measure space $(X, \mathcal{A}, \mu)$, and let $\tau$ be an inducing time (mod $\mu$ ) for $T$ on $Y \in \mathcal{A}^{+}(\mu)$. Suppose that $T^{\tau}$ has a finite invariant measure $\nu \ll \mu$ on $(Y, \mathcal{A} \cap Y)$. Then either

$$
\int_{Y} \tau d \nu<\infty \quad \text { and } \quad \mu=\kappa \cdot\left(\tau \times_{T} \nu\right) \text { for some } \kappa>0
$$

or

$$
\int_{Y} \tau d \nu=\infty \quad \text { and } \quad\left(\tau \times_{T} \nu\right)(E)=\infty \text { for all } E \in \mathcal{A}^{+}(\mu) .
$$

Proof. Assume that $\int_{Y} \tau d \nu<\infty$. By Proposition 1.1 $\tau \times_{T} \nu \ll \mu$ is then a finite invariant measure for $T$, hence $\tau \times_{T} \nu=\kappa^{-1} \mu$ for some $\kappa>0$ by ergodicity. Suppose then that $\int_{Y} \tau d \nu=\infty$. Since $\tau \times_{T} \nu \ll \mu$ and $\bigcup_{n \geq 0} T^{-n} E=X \bmod \mu$ for all $E \in \mathcal{A}^{+}(\mu)$, we have $0<\nu(Y) \leq\left(\tau \times_{T} \nu\right)(X) \leq \sum_{n \geq 0}\left(\tau \times_{T} \nu\right)\left(T^{-n} E\right)$, so that $\left(\tau \times \times_{T} \nu\right)(E)>0$ for all $E \in \mathcal{A}^{+}(\mu)$ due to the $T$-invariance of this measure. If there is an $E \in \mathcal{A}^{+}(\mu)$ with $\left(\tau \times_{T} \nu\right)(E)<\infty$, we consider the induced map $T_{E}: E \rightarrow E$ which preserves the two finite measures $\tau \times\left.\left._{T} \nu\right|_{\mathcal{A} \cap E} \ll \mu\right|_{\mathcal{A} \cap E}$. The second one being ergodic, we conclude that $\tau \times\left._{T} \nu\right|_{\mathcal{A} \cap E}=\left.\kappa^{-1} \mu\right|_{\mathcal{A} \cap E}$ and hence $\tau \times_{T} \nu=\kappa^{-1} \mu$ for some $\kappa>0$, contradicting $\left(\tau \times_{T} \nu\right)(X)=\infty$. 
While this lemma characterizes all possibilities if $T^{\tau}$ is known to have an a.c.i.m. $\nu$, the question of whether or not such a measure $\nu$ exists is more intricate. The most extreme (and most obvious) way in which $T^{\tau}$ can fail to have an invariant measure $\nu$ with $\mu=\tau \times_{T} \nu$ is by being totally dissipative and admitting no a.c.i.m. at all:

Example 2.1 (A totally dissipative inducing time). Consider $X:=\{0,1\}^{\mathbb{N}_{0}}$ with $\sigma$-field $\mathcal{A}$ generated by the collection of cylinders of order $n \in \mathbb{N}$, i.e. of the sets $\left[i_{0}, \ldots, i_{n-1}\right]:=\left\{x=\left(x_{j}\right)_{j \in \mathbb{N}_{0}} \in X: x_{j}=i_{j}\right.$ for $\left.j \in\{0, \ldots, n-1\}\right\}$, and Bernoulli $\left(\frac{1}{2}, \frac{1}{2}\right)$-product measure $\mu$. The shift transformation $T,(T x)_{j}=x_{j+1}$ is an ergodic m.p.t. on $(X, \mathcal{A}, \mu)$. Define $\tau: X \rightarrow \mathbb{N}$ by letting

$$
\tau:=\varphi_{[\underbrace{0, \ldots, 0}_{m+1}]}^{0, \ldots}[\underbrace{0, \ldots, 0}_{m}, 1], m \in \mathbb{N}_{0} .
$$

Then $T^{\tau}$ is easily seen to be totally dissipative, and there is no $T^{\tau}$-invariant measure $\nu \ll \mu$ at all. Notice also that $\tau$ has infinite $\mu$-expectation: $\int_{X} \tau d \mu=\infty$.

However, it can also happen that $T^{\tau}$ preserves the same finite measure $\mu=: \nu$ as $T$, hence is conservative, and still does not satisfy $\mu=\tau \times_{T} \nu$ up to a constant factor, since $\int_{Y} \tau d \nu=\infty$ (recall Lemma 2.1):

Example 2.2 (A situation in which $\mu \neq \tau \times_{T} \nu$ ). Let $(X, \mathcal{A}, \mu), T$, and $\xi_{n}$ be as in Example 2.1. To any $(\bmod \mu)$ partition $\zeta \subseteq \bigcup_{n>1} \xi_{n}$ of $X$ into cylinder sets, we associate an inducing time $\tau_{\zeta}$ for $T$ on $X$ defined by

$$
\tau_{\zeta}:=n \quad \text { on } Z \in \zeta \cap \xi_{n}, n \geq 1 \text {. }
$$

Then it is easy to check that $T^{\tau_{\zeta}}: X \rightarrow X$ corresponds to a full shift over the alphabet $\zeta$, and hence again preserves $\mu=: \nu$ and is ergodic. Now $\int_{X} \tau_{\zeta} d \nu=$ $\int_{X} \tau_{\zeta} d \mu$, but obviously there are many $\zeta$ 's for which $\int_{X} \tau_{\zeta} d \mu=\infty$.

The fact that in both counterexamples above $\tau$ has infinite $\mu$-expectation is consistent with Theorem 1.1 On the other hand, $\mu$-integrability of $\tau$ is not a necessary condition for the existence of such an invariant measure. In fact, $\tau \times_{T} \nu$ may assign much more mass to sets $\{\tau>n\}$ than $\nu$ does:

Example 2.3 (Good inducing times need not be $\mu$-integrable). We illustrate this phenomenon by means of discrete renewal Markov chains. Let $X:=\mathbb{N}_{0}^{\mathbb{N}_{0}}=\{x=$ $\left.\left(x_{j}\right)_{j \in \mathbb{N}_{0}}: x_{j} \in \mathbb{N}_{0}\right\}$, let $\xi_{n}$ be the collection of cylinders $\left[i_{0}, \ldots, i_{n-1}\right]:=\{x=$ $\left(x_{j}\right)_{j \in \mathbb{N}_{0}} \in X: x_{j}=i_{j}$ for $\left.j \in\{0, \ldots, n-1\}\right\}$ of order $n \in \mathbb{N}$, and let $\mathcal{A}$ be the $\sigma$-field generated by the $\xi_{n}$. Fix $\alpha>1$, let $f_{k}:=k^{-(1+\alpha)} /\left(\sum_{k \geq 1} k^{-(1+\alpha)}\right)$, $k \in \mathbb{N}$, and define transition probabilities on $\mathbb{N}_{0}$ by $p_{0, k}:=f_{k}$ and $p_{k+1, k}:=1$, $k \geq 0$. Then the shift transformation $T$ preserves a Markov probability measure $\mu$ on $(X, \mathcal{A})$ with $\mu([i])=\left(\sum_{k>i} f_{k}\right) /\left(\sum_{k \geq 1} k f_{k}\right), i \in \mathbb{N}_{0}$. Consider the inducing time $\tau$ on $X$ given by $\left.\tau\right|_{[i]}:=i+1$. Then $T^{\tau}$ corresponds to a full shift over the alphabet $\zeta:=\left\{[i]: i \in \mathbb{N}_{0}\right\}$, and it preserves the Bernoulli product measure $\nu \ll \mu$ on $X$ with $\nu([0])=\mu([0])$ and $\nu([i])=f_{i} \mu(X \backslash[0]), i \geq 1$. It is easy to verify that $\int_{X} \tau d v<\infty$ (and hence $\mu=$ const $\cdot \tau \times_{T} \nu$ ) for all $\alpha>1$, while $\int_{X} \tau d \mu<\infty$ iff $\alpha>2$. 


\section{INTEGRABLE INDUCING TIMES}

Our proof of Theorem 1.1 depends on the following construction: Let $T$ be a nonsingular transformation on the $\sigma$-finite measure space $(X, \mathcal{A}, m)$, and let $\tau$ be an inducing time $(\bmod m)$ for $T$ on $Y \in \mathcal{A}^{+}(m)$. The $\tau$-separating extension of $(X, \mathcal{A}, m, T)$ is the nonsingular transformation $(\widehat{X}, \widehat{\mathcal{A}}, \widehat{m}, \widehat{T})$ obtained as follows:

Extend the definition of $\tau$ to all of $X$ by letting $\tau(x):=0$ for $x \in Y^{c}$. The extension $\widehat{T}$ of $T$ we are going to construct will act on a space $\widehat{X} \subseteq X \times \mathbb{N}_{0} \times \mathbb{N}_{0}$, with natural projections given by $\pi(\widehat{x}):=x, \widehat{\tau}(\widehat{x}):=t$, and $\Lambda(\widehat{x}):=l$ for $\widehat{x}=(x, t, l)$. It becomes a $\sigma$-finite measure space $(\widehat{X}, \widehat{\mathcal{A}}, \widehat{m})$ if we regard it as a subspace of $X \times \mathbb{N}_{0} \times \mathbb{N}_{0}$ with product ( $\sigma$-field and) measure $m \otimes \iota \otimes \iota$, where $\iota$ denotes counting measure on $\mathbb{N}_{0}$. We inductively define the level sets $\widehat{X}_{l}=\widehat{X} \cap\{\Lambda=l\}, l \geq 0$, of the extension: Let $\widehat{\mathcal{M}}_{0}:=\left\{\{\tau=t\} \times\{t\} \times\{0\}: t \in \mathbb{N}_{0}\right\}$, and $\widehat{X}_{0}:=\bigcup \widehat{\mathcal{M}}_{0}$. Given $\widehat{X}_{l}=\bigcup \widehat{\mathcal{M}}_{l}$, we define $\widehat{\mathcal{M}}_{l+1}:=\left\{T E \times\{t\} \times\{l+1\}: \emptyset \neq E \times\{t\} \times\{l\} \in \widehat{\mathcal{M}}_{l}\right.$ with $l<t-1\}$, and let $\widehat{X}_{l+1}:=\bigcup \widehat{\mathcal{M}}_{l+1}$. This determines $\widehat{X}:=\bigcup_{l>0} \widehat{X}_{l}=\bigcup \widehat{\mathcal{M}}$, where $\widehat{\mathcal{M}}:=\bigcup_{l \geq 0} \widehat{\mathcal{M}}_{l}$. Notice that $\Lambda<\widehat{\tau}$ on $\widehat{X} \backslash\left(Y^{c} \times\{0\} \times\{0\}\right)$. The map $\widehat{T}: \widehat{X} \rightarrow \widehat{X}$ is given by

$$
\widehat{T}(x, t, l):= \begin{cases}(T x, t, l+1) & \text { if } l<t-1 \\ (T x, \tau(T x), 0) & \text { otherwise }\end{cases}
$$

which clearly is nonsingular on $(\widehat{X}, \widehat{\mathcal{A}}, \widehat{m})$. We have

$$
\Lambda(\widehat{T}(x, t, l))= \begin{cases}l+1 & \text { if } l<t-1 \\ 0 & \text { otherwise }\end{cases}
$$

and $\pi: \widehat{X} \rightarrow X$ is a factor map, i.e. $\pi \circ \widehat{T}=T \circ \pi$ on $\widehat{X}$. This projection also provides us with natural isomorphisms $\widehat{X}_{0} \cong X$, and $\widehat{Y}_{0}:=\widehat{X}_{0} \cap \pi^{-1} Y \cong Y$. Of course, $\widehat{Y}:=\bigcup_{n \geq 0} \widehat{T}^{n} \widehat{Y}_{0}$ is forward invariant under $\widehat{T}$, and we also see that $\widehat{Y}_{0}$ is a sweep-out set for $\widehat{Y}$, that is, $\widehat{Y} \subseteq \bigcup_{n \geq 1} \widehat{T}^{-n} \widehat{Y}_{0} \bmod \widehat{\mu}$. In fact, $\left.\widehat{\tau}\right|_{\widehat{Y}_{0}}=\left.\tau \circ \pi\right|_{\widehat{Y}_{0}}$ almost surely equals the first return time $\widehat{\varphi}_{\widehat{Y}_{0}}$ of $\widehat{Y}_{0}$ under $\widehat{T}$, so that

$$
\left(Y, \mathcal{A} \cap Y, m, T^{\tau}\right) \cong\left(\widehat{Y}_{0}, \widehat{\mathcal{A}} \cap \widehat{Y}_{0},\left.\widehat{m}\right|_{\widehat{\mathcal{A}} \cap \widehat{Y}_{0}}, \widehat{T}_{\widehat{Y}_{0}}\right)
$$

as nonsingular transformations via the identification by $\left.\pi\right|_{\widehat{Y}_{0}}$. Therefore,

$$
\begin{gathered}
\text { existence of a finite invariant measure } \nu \ll m \text { for } T^{\tau} \\
\text { is equivalent to the existence of some } \widehat{T} \text {-invariant } \\
\sigma \text {-finite measure } \widehat{\nu} \ll \widehat{m} \text { on } \widehat{Y} \text { with } \widehat{\nu}\left(\widehat{Y}_{0}\right)<\infty \text {. }
\end{gathered}
$$

(As $\widehat{Y}_{0}$ is a sweep-out set, we must have $\widehat{\nu}\left(\widehat{Y}_{0}\right)>0$ in this case, and hence may take $\nu(E):=\widehat{\nu}\left(\widehat{Y}_{0} \cap \pi^{-1} E\right), E \in \mathcal{A} \cap Y$.) Notice also that the image measure $\widehat{\nu} \circ \pi^{-1}$ is always an absolutely continuous invariant measure for $T$ in this case (not necessarily $\sigma$-finite).

We let $\mathcal{L}$ and $\widehat{\mathcal{L}}$ denote the dual operators of $T$ and $\widehat{T}$ (w.r.t. $m$ and $\widehat{m}$ ) respectively, i.e. $\mathcal{L}$ is the positive linear operator on $L_{1}(X, \mathcal{A}, m)$ (with an obvious extension to all nonnegative measurable functions) characterized by

$$
\int_{X} \mathcal{L} u \cdot g d m=\int_{X} u \cdot g \circ T d m \quad \text { for } u \in L_{1}(X, \mathcal{A}, m), g \in L_{\infty}(X, \mathcal{A}, m),
$$


and analogously for $\widehat{\mathcal{L}}$. Then $\pi_{*} \circ \widehat{\mathcal{L}}=\mathcal{L} \circ \pi_{*}$, where $\pi_{*}$ is the dual of $\pi$, that is, $\pi_{*}(\widehat{u})(x):=\sum_{\widehat{x} \in \pi^{-1}(x)} \widehat{u}(\widehat{x})$. A measurable function $\widehat{h}: \widehat{X} \rightarrow[0, \infty]$ vanishing outside $\widehat{Y}$ is the density of some $\widehat{T}$-invariant measure $\widehat{\nu} \ll \widehat{m}$ on $\widehat{Y}$ iff $\widehat{\mathcal{L}} \widehat{h}=\widehat{h}$. In this case we have

$$
1_{\widehat{E}} \widehat{h}=\widehat{\mathcal{L}}^{l}\left(1_{\left(\{\tau=t\} \cap T^{-l} E\right) \times\{t\} \times\{0\}} \cdot \widehat{h}\right) \quad \text { for } \widehat{E}=E \times\{t\} \times\{l\} \in \widehat{\mathcal{M}} \text { with } l \geq 1,
$$

since $(\widehat{T})^{-l} \widehat{E}=\left(\{\tau=t\} \cap T^{-l} E\right) \times\{t\} \times\{0\}$. Projecting $\widehat{h}=\sum_{\widehat{E} \in \widehat{M}} 1_{\widehat{E}} \widehat{h}$ onto $X$, we find that

if $\widehat{\nu} \ll \widehat{m}$ is $\widehat{T}$-invariant, then $\widehat{\nu} \circ \pi^{-1}=\tau \times_{T} \nu$, where $\nu:=\left.\widehat{\nu}\right|_{\widehat{Y}_{0}} \circ \pi^{-1} \ll m$ is $T^{\tau}$-invariant.

We are now in a position to prove that $\int_{Y} \tau d \mu<\infty$ is sufficient:

Proof of Theorem 1.1. Assume w.l.o.g. that $\mu(Y)=1$, and let $(\widehat{X}, \widehat{\mathcal{A}}, \widehat{\mu}, \widehat{T})$ be the $\tau$-separating extension of $(X, \mathcal{A}, \mu, T)$. Due to ergodicity of $T$, 3.3) together with our previous observations implies that

$$
\begin{aligned}
\mu=\tau \times_{T} \nu \text { has a solution } & \Longleftrightarrow \mu \text { lifts to an a.c.i.m. of }\left.\widehat{T}\right|_{\widehat{Y}} \\
& \left.\Longleftrightarrow \widehat{T}\right|_{\widehat{Y}} \text { has a finite a.c.i.m. }
\end{aligned}
$$

(i) Iterating densities on the extension. We would like to obtain an invariant probability density $\widehat{h}=\widehat{\mathcal{L}} \widehat{h}$ for $\widehat{T}$ on $\widehat{Y}$ as an accumulation point of the sequence of averages $\widehat{A}_{n}:=n^{-1} \sum_{k=0}^{n-1} \widehat{\mathcal{L}}^{k} 1_{\widehat{Y}_{0}}: \widehat{X} \rightarrow[0,1], n \geq 1$ (which vanish outside $\widehat{Y}$ ). Since $\mu$ is $T$-invariant, we have $1_{X}=\mathcal{L}^{k} 1_{X}=\pi_{*}\left(\widehat{\mathcal{L}}^{k} 1_{\widehat{X}_{0}}\right)$ for $k \geq 0$, and hence

$$
0 \leq \widehat{\mathcal{L}}^{k} 1_{\widehat{Y}_{0}} \leq 1_{\widehat{X}} \quad \text { for } k \geq 0 .
$$

Since, for finite measure spaces, weak sequential precompactness in $L_{1}$ is equivalent to uniform integrability (cf. [DS], corollary IV.8.11), [3.5) implies that for any $\widehat{E} \subseteq \widehat{X}$ which is bounded in the sense that $\widehat{\tau}(\widehat{E})$ and $\Lambda(\widehat{E})$ are bounded subsets of $\mathbb{N}_{0}$

$$
\left(\left.\widehat{A}_{n}\right|_{\widehat{E}}\right)_{n \geq 1} \text { is weakly sequentially precompact in } L_{1}\left(\widehat{E}, \widehat{\mathcal{A}} \cap \widehat{E},\left.\widehat{\mu}\right|_{\widehat{\mathcal{A}} \cap \widehat{E}}\right) \text {. }
$$

As $\widehat{X}$ is the union of an increasing sequence of bounded sets, a straightforward diagonalization argument shows that there are $n_{k} \nearrow \infty$ and some measurable $\widehat{h}: \widehat{X} \rightarrow[0,1]$, vanishing outside $\widehat{Y}$, such that for all bounded $\widehat{E} \in \widehat{\mathcal{A}}$,

$$
\left.\left.\widehat{A}_{n_{k}}\right|_{\widehat{E}} \underset{k \rightarrow \infty}{\text { weakly in } L_{1}\left(\widehat{E}, \widehat{\mathcal{A}} \cap \widehat{E},\left.\widehat{\mu}\right|_{\widehat{\mathcal{A}} \cap \widehat{E}}\right)} \widehat{h}\right|_{\widehat{E}} \quad \text { for all bounded } \widehat{E} \in \widehat{\mathcal{A}} \text {. }
$$

Let $\widehat{\nu}$ denote the $\sigma$-finite measure with density $\widehat{h}$ on $(\widehat{X}, \widehat{\mathcal{A}}, \widehat{\mu})$. According to (3.6) we have

$$
\widehat{\nu}(\widehat{E})=\lim _{k \rightarrow \infty} \int_{\widehat{E}} \widehat{A}_{n_{k}} d \widehat{\mu} \quad \text { for all bounded } \widehat{E} \in \widehat{\mathcal{A}} .
$$

Recall that $\int_{\widehat{X}} 1_{\widehat{Y}_{0}} d \widehat{\mu}=\mu(Y)=1$ by assumption, so that the $\widehat{A}_{n}$ are probability densities. Hence we see that $\widehat{\nu}(\widehat{X}) \leq 1$. However, we might have $\widehat{\nu} \equiv 0$.

(ii) Obtaining an invariant density if $\int_{X} \tau d \mu<\infty$. To see that $\widehat{\nu}$ is a nonvanishing invariant measure for $\widehat{T}$, we need some control of how much mass will be pushed to sets $E \times\{t\} \times\{l\} \in \widehat{\mathcal{M}}$ with large $t$ and $l$ if we start with the initial 
density $1_{\widehat{Y}_{0}}$ on $\widehat{X}$ and iterate $\widehat{\mathcal{L}}$. Because of $\Lambda<\widehat{\tau}$ on $\widehat{Y}$, and (3.1) we see that for any $t, l, k \in \mathbb{N}_{0}$,

$\widehat{\mathcal{L}}^{k} 1_{\widehat{Y}_{0}}=\left\{\begin{array}{ll}\widehat{\mathcal{L}}^{l} & \left(1_{\widehat{X}_{0} \cap\{\widehat{\tau}=t\}} \cdot \widehat{\mathcal{L}}^{k-l} 1_{\widehat{Y}_{0}}\right) \\ 0 & \begin{array}{l}\text { if } k \geq l \text { and } t>l \\ \text { otherwise }\end{array}\end{array}\right\}$ on $\widehat{X} \cap\{\widehat{\tau}=t\} \cap\{\Lambda=l\}$, and therefore, recalling (3.5) and $\widehat{\tau}=\tau \circ \pi$ on $\widehat{X}_{0}$,

$$
\begin{aligned}
\int_{\widehat{X} \cap\{\widehat{\tau}>t \text { and } \Lambda=l\}} \widehat{\mathcal{L}}^{k} 1_{\widehat{Y}_{0}} d \widehat{\mu} & = \begin{cases}\int_{\widehat{X}_{0} \cap\{\widehat{\tau}>t \vee l\}} \widehat{\mathcal{L}}^{k-l} 1_{\widehat{Y}_{0}} d \widehat{\mu} & \text { if } k \geq l, \\
0 & \text { otherwise }\end{cases} \\
& \leq \begin{cases}\mu(\{\tau>t \vee l\}) & \text { if } k \geq l, \\
0 & \text { otherwise. }\end{cases}
\end{aligned}
$$

Decomposing $\widehat{X} \cap\{\widehat{\tau}>t\}=\bigcup_{l \geq 0} \widehat{X} \cap\{\widehat{\tau}>t$ and $\Lambda=l\}$, we end up with

$$
\int_{\widehat{X} \cap\{\widehat{\tau}>t\}} \widehat{\mathcal{L}}^{k} 1_{\widehat{Y}_{0}} d \widehat{\mu} \leq \sum_{l=0}^{k} \mu(\{\tau>t \vee l\})=\sum_{l=t}^{k} \mu(\{\tau>l\})+t \cdot \mu(\{\tau>t\}) .
$$

Since, by assumption, $\int_{X} \tau d \mu<\infty$, we conclude that there is some sequence $\left(t_{j}\right)_{j \geq 1}$ in $\mathbb{N}$ such that $t_{j} \nearrow \infty$ and

$$
\int_{\widehat{X} \cap\left\{\widehat{\tau}>t_{j}\right\}} \widehat{\mathcal{L}}^{k} 1_{\widehat{Y}_{0}} d \widehat{\mu} \leq \frac{1}{j} \quad \text { for all } j \in \mathbb{N}, k \in \mathbb{N}_{0} .
$$

Now consider the bounded sets $\widehat{D}_{j}:=\bigcup \widehat{\mathcal{D}}_{j}$, where each $\widehat{\mathcal{D}}_{j}:=\{E \times\{t\} \times\{l\} \in$ $\left.\widehat{\mathcal{M}}: t \leq t_{j}\right\}, j \geq 1$, is a finite collection, and $\widehat{D}_{j} \nearrow \widehat{X}$. Since (3.10) ensures that $\int_{\widehat{D}_{j}^{c}} \widehat{A}_{n} d \widehat{\mu}<\frac{1}{j}$ for all $n, j \in \mathbb{N}$, we see that $\widehat{\nu}(\widehat{X})=1$ and $\widehat{\nu}\left(\widehat{D}_{j}^{c}\right) \leq \frac{1}{j}$ for all $j$. Observe next that (3.7) remains true if we replace $n_{k}$ by $n_{k}+1$, and that $\int_{\widehat{T}^{-1} \widehat{E}} \widehat{A}_{n} d \widehat{\mu}=\frac{n+1}{n} \int_{\widehat{E}} \widehat{A}_{n+1} d \widehat{\mu}-\frac{1}{n} \int_{\widehat{E}} 1_{\widehat{Y}_{0}} d \widehat{\mu}$ for all $n \geq 1$ and $\widehat{E} \in \widehat{\mathcal{A}}$. Fix any bounded $\widehat{E}$ and $j \in \mathbb{N}$. Then

$$
\begin{aligned}
\widehat{\nu}\left(\widehat{T}^{-1} \widehat{E}\right) & =\widehat{\nu}\left(\widehat{T}^{-1} \widehat{E} \cap \widehat{D}_{j}\right)+\delta_{1}=\lim _{k \rightarrow \infty} \int_{\widehat{T}^{-1} \widehat{E} \cap \widehat{D}_{j}} \widehat{A}_{n_{k}} d \widehat{\mu}+\delta_{1} \\
& =\lim _{k \rightarrow \infty} \int_{\widehat{T}^{-1} \widehat{E}} \widehat{A}_{n_{k}} d \widehat{\mu}+\delta_{1}+\delta_{2} \\
& =\lim _{k \rightarrow \infty} \int_{\widehat{E}} \widehat{A}_{n_{k}+1} d \widehat{\mu}+\delta_{1}+\delta_{2}=\widehat{\nu}(\widehat{E})+\delta_{1}+\delta_{2}
\end{aligned}
$$

with $\left|\delta_{i}\right| \leq \frac{1}{j}$, which implies $\widehat{T}$-invariance of $\widehat{\nu}$.

Remark 3.1. The concept of the $\tau$-separating extension was inspired by lifting results for Markov extensions; cf. [Ke]. Parts of our argument can also be found there. The idea of representing certain inducing times as first-return times of an extension also appears (in the special setup of interval maps and Markov extensions) e.g. in $[\mathrm{Br}]$.

\section{A COMPlete Characterization}

We turn to a complete characterization of those inducing times $\tau: Y \rightarrow \overline{\mathbb{N}}$ for a conservative ergodic m.p.t. on a $\sigma$-finite measure space $(X, \mathcal{A}, \mu)$ for which $\mu=\tau \times_{T} \nu$ has a solution. The proof of Theorem 1.2 and the subsequent short 
discussion of infinite measure-preserving situations depend on the following (hardly surprising) observation.

Lemma 4.1 (Chain rule for $\times_{T}$ ). Let $T$ be a nonsingular transformation on $(X, \mathcal{A}, m)$. Consider $Y \subseteq Y^{\prime}$ in $\mathcal{A}^{+}(\mu)$, and assume that $\tau$ and $\psi$ respectively are inducing times for $T$ on $Y$ and $Y^{\prime}$ such that $\tau=\psi_{\rho}:=\sum_{k=0}^{\rho-1} \psi \circ\left(T^{\psi}\right)^{k}$ for some inducing time $\rho$ for $T^{\psi}$ on $Y$, i.e. $T^{\tau}=\left(T^{\psi}\right)^{\rho}$. If $\nu \ll m$ is a measure on $(Y, \mathcal{A} \cap Y)$, then

$$
\tau \times_{T} \nu=\psi \times_{T}\left(\rho \times_{T} \psi \nu\right) .
$$

Proof. Fix $E \in \mathcal{A}$. As $l \mapsto \psi_{l}$ is strictly increasing, we have $\{\rho>l\}=\left\{\tau>\psi_{l}\right\}$, and hence

$$
\begin{aligned}
& \psi \times_{T}\left(\rho \times_{T^{\psi}} \nu\right)(E) \\
& =\sum_{m \geq 0}\left(\rho \times_{T^{\psi}} \nu\right)\left(\{\psi>m\} \cap T^{-m} E\right) \\
& =\sum_{m, l \geq 0} \nu\left(\{\rho>l\} \cap\left(T^{\psi}\right)^{-l}\left(\{\psi>m\} \cap T^{-m} E\right)\right) \\
& =\sum_{m, l, r \geq 0} \nu\left(\{\rho>l\} \cap\left\{\psi_{l}=r\right\} \cap T^{-r}\left(\{\psi>m\} \cap T^{-m} E\right)\right) \\
& =\sum_{m, l, r \geq 0} \nu\left(\{\tau>r\} \cap\left\{\psi_{l}=r\right\} \cap T^{-r}\{\psi>m\} \cap T^{-(m+r)} E\right) .
\end{aligned}
$$

Let $L(i):=\max \left\{l \geq 0: \psi_{l} \leq i\right\}$ and $R(i):=\psi_{L(i)}, i \geq 0$. Then, for any $t \in \mathbb{N}$,

$$
\begin{aligned}
& \sum_{m, l \geq 0} \sum_{0 \leq r<t} \nu\left(\{\tau=t\} \cap\left\{\psi_{l}=r\right\} \cap T^{-r}\{\psi>m\} \cap T^{-(m+r)} E\right) \\
& =\sum_{m, l \geq 0} \sum_{0 \leq r<t} \nu\left(\{\tau=t\} \cap\left\{r=\psi_{l} \leq m+r<\psi_{l+1}\right\} \cap T^{-(m+r)} E\right) \\
& =\sum_{m \geq 0} \sum_{0 \leq r<t} \nu\left(\{\tau=t\} \cap\{R(m+r)=r\} \cap T^{-(m+r)} E\right) \\
& =\sum_{i \geq 0} \sum_{0 \leq r<t} \nu\left(\{\tau=t\} \cap\{R(i)=r\} \cap T^{-i} E\right) \\
& =\sum_{0 \leq i<t} \nu\left(\{\tau=t\} \cap T^{-i} E\right),
\end{aligned}
$$

where the last step uses the fact that $i<t$ on $\left\{\psi_{L(i)}=R(i)=r<t=\tau=\psi_{\rho}\right\}$. Since $\left(\tau \times_{T} \nu\right)(E)=\sum_{t \geq 1} \sum_{0 \leq i<t} \nu\left(\{\tau=t\} \cap T^{-i} E\right)$, the proof is complete.

Remark 4.1. If, more specifically, $\nu \ll m$ is invariant for $T^{\tau}$, and one of $\tau \times_{T} \nu$ and $\psi \times_{T}\left(\rho \times_{T \psi} \nu\right)$ is known to be finite, an alternative quick proof of the chain rule is as follows: According to Proposition 1.1 both measures are a.c.i.m.s. for T. By ergodicity it is therefore enough to show that they have the same total mass. But $\left(\psi \times_{T}\left(\rho \times_{T^{\psi}} \nu\right)\right)(X)=\int \psi d\left(\rho \times_{T} \psi \nu\right)=\int \sum_{n \geq 0} 1_{\{\rho>n\}}\left(\psi \circ\left(T^{\psi}\right)^{n}\right) d \nu=\int \tau d \nu=$ $\left(\tau \times_{T} \nu\right)(X)$ by Proposition 1.1 .

Proof of Theorem 1.2. Note that for any $Z \in \mathcal{A}^{+}(\mu)$ with $Z \subseteq Y, \theta:=\theta(\tau, Z)$ is an inducing time $(\bmod \mu)$ for $T$ on $Z$ satisfying $\left(T^{\tau}\right)_{Z}=T^{\theta}$. 
(i) Assume that $\int_{Z} \theta d \mu<\infty$. By Theorem 1.1 there is some invariant measure $\left.\nu\right|_{Z} \ll \mu$ on $Z$ for $T^{\theta}$ for which $\mu=\theta \times_{T}\left(\left.\nu\right|_{\mathcal{A} \cap Z}\right)$. Extend $\left.\nu\right|_{\mathcal{A} \cap Z}$ to a $T^{\tau}$-invariant measure $\nu \ll \mu$ on $(Y, \mathcal{A} \cap Y)$ by letting $\nu:=\varphi_{Z}^{\tau} \times_{T^{\tau}}\left(\left.\nu\right|_{\mathcal{A} \cap Z}\right)$. According to the chain rule,

$$
\mu=\theta \times_{T}\left(\left.\nu\right|_{\mathcal{A} \cap Z}\right)=\tau \times_{T}\left(\varphi_{Z}^{\tau} \times_{T^{\tau}}\left(\left.\nu\right|_{\mathcal{A} \cap Z}\right)\right)=\tau \times_{T} \nu .
$$

(ii) Conversely, assume that $\mu=\tau \times_{T} \nu$ has a solution $\nu$. Take $Z \subseteq Y$ with $\nu(Z)>0$. Since $\nu=\varphi_{Z}^{\tau} \times_{T^{\tau}}\left(\left.\nu\right|_{\mathcal{A} \cap Z}\right)$, we have

$$
\mu=\tau \times_{T} \nu=\tau \times_{T}\left(\varphi_{Z}^{\tau} \times_{T^{\tau}}\left(\left.\nu\right|_{\mathcal{A} \cap Z}\right)\right),
$$

so that the chain rule applies to show that $\mu=\theta \times_{T}\left(\left.\nu\right|_{\mathcal{A} \cap Z}\right)$. In particular, $\int_{Z} \theta d \nu=\mu(X)<\infty$. Now $\left.\left.\mu\right|_{\mathcal{A} \cap \tilde{Y}} \ll \nu\right|_{\mathcal{A} \cap \tilde{Y}}$, where $\tilde{Y}:=\left\{\frac{d \nu}{d \mu}>0\right\} \subseteq Y$, and if we take any $Z \in \mathcal{Z}(\nu):=\left\{E \in \mathcal{A}^{+}(\mu): \operatorname{essinf}_{E} \frac{d \nu}{d \mu}>0\right\}$, we have $\int_{Z} \theta d \mu<\infty$.

If $T$ preserves an infinite measure $\mu$ in the first place (cf. [A0]), it is clear that we cannot directly use integrability arguments, since for any $Y$ with $\mu(Y)<\infty$ we have $\int_{Y} \varphi_{Y} d \mu=\infty$ and hence $\int_{Y} \tau d \mu=\infty$ for any inducing time $\tau$ on $Y$. Instead, we show that we can always pass to first return maps on arbitrary subsets $Z \subseteq Y$ without losing any information. Choosing $Z$ to have finite measure, the following proposition together with Theorem 1.2 yields a complete answer to our question, even in the $\mu(X)=\infty$ case.

In order to state it, observe that if $T$ is a c.e.m.p.t. on the $\sigma$-finite measure space $(X, \mathcal{A}, \mu), \tau$ an inducing time for $T$ on $Y \in \mathcal{A}^{+}(\mu)$, and $Z \in \mathcal{A}^{+}(\mu) \cap Y$, then the first return map of $T^{\tau}$ to $Z$ can be represented as $\left(T^{\tau}\right)_{Z}=\left(T_{Z}\right)^{\vartheta}$ with $\vartheta: Z \rightarrow \mathbb{N}$ an inducing time for $T_{Z}$.

Proposition 4.1 (Passing to first return maps). Let $T$ be a c.e.m.p.t. on the $\sigma$ finite measure space $(X, \mathcal{A}, \mu)$, and let $\tau$ be an inducing time for $T$ on $Y \in \mathcal{A}^{+}(\mu)$. Suppose that $Z \in \mathcal{A}^{+}(\mu) \cap Y$ with $\left(T^{\tau}\right)_{Z}=\left(T_{Z}\right)^{\vartheta}$. Then

$$
\mu=\tau \times_{T} \nu \quad \text { has a solution } \nu
$$

iff

$$
\left.\mu\right|_{\mathcal{A} \cap Z}=\vartheta \times_{T_{Z}} \widetilde{\nu} \quad \text { has a solution } \widetilde{\nu} .
$$

In this case, $\nu=\varphi_{Z}^{\tau} \times_{T^{\tau}} \widetilde{\nu}$, that is, $\widetilde{\nu}=\left.\nu\right|_{\mathcal{A} \cap Z}$.

Proof. Suppose that there is some measure $\widetilde{\nu}$ on $(Z, \mathcal{A} \cap Z)$ satisfying $\left.\mu\right|_{\mathcal{A} \cap Z}=$ $\vartheta \times_{T_{Z}} \widetilde{\nu}$. Then $\widetilde{\nu} \leq\left.\mu\right|_{\mathcal{A} \cap Z}$ is a $\sigma$-finite invariant measure for $\left(T^{\tau}\right)_{Z}=\left(T_{Z}\right)^{\vartheta}$, hence $\nu:=\varphi_{Z}^{\tau} \times_{T^{\tau}} \widetilde{\nu}$ is a $\sigma$-finite invariant measure for $T^{\tau}$. The chain rule implies that

$$
\begin{aligned}
\mu & =\varphi_{Z} \times\left._{T} \mu\right|_{\mathcal{A} \cap Z}=\varphi_{Z} \times_{T}\left(\vartheta \times_{T_{Z}} \widetilde{\nu}\right) \\
& =\tau \times_{T}\left(\varphi_{Z}^{\tau} \times_{T^{\tau}} \widetilde{\nu}\right)=\tau \times_{T} \nu .
\end{aligned}
$$

Conversely, suppose that $\mu=\tau \times_{T} \nu$ for some measure $\nu$ on $(Y, \mathcal{A} \cap Y)$. By the chain rule again, $\mu=\tau \times_{T} \nu=\tau \times_{T}\left(\varphi_{Z}^{\tau} \times\left._{T^{\tau}} \nu\right|_{\mathcal{A} \cap Z}\right)=\varphi_{Z} \times_{T}\left(\vartheta \times\left._{T_{Z}} \nu\right|_{\mathcal{A} \cap Z}\right)$. According to Remark 2.1, this implies that $\left.\mu\right|_{\mathcal{A} \cap Z}=\vartheta \times\left._{T_{Z}} \nu\right|_{\mathcal{A} \cap Z}$ since $T$ is a c.e.m.p.t.

We conclude with another observation of similar flavour.

Remark 4.2 (Factorizing through first-return maps). Let $T$ be a nonsingular transformation on the $\sigma$-finite measure space $(X, \mathcal{A}, \mu)$, and let $\tau$ be an inducing time for $T$ on $Y \in \mathcal{A}^{+}(\mu)$. Notice that for a.e. $x \in Y, \tau(x)$ has to be one of the successive 
return times $\varphi_{Y, n}:=\sum_{k=0}^{n-1} \varphi_{Y} \circ T_{Y}^{k}, n \geq 1$, to this set, i.e. $\tau=\varphi_{Y, \rho}$ a.e. for some $\rho: Y \rightarrow \mathbb{N}$ which is measurable since $\{\rho=k\}=\left\{\tau=\varphi_{Y, k}\right\}$. Hence $T^{\tau}$ factorizes through $T_{Y}$ in that $T^{\tau}=\left(T_{Y}\right)^{\rho}$. The chain rule and (1.3) are easily seen to imply that for any measure $\nu$ on $(Y, \mathcal{A} \cap Y)$,

$$
\mu=\tau \times_{T} \nu \quad \text { iff }\left.\quad \mu\right|_{\mathcal{A} \cap Y}=\rho \times_{T_{Y}} \nu .
$$

Observe that, via Theorem 1.1, this shows that

$$
\int_{Y} \rho d \mu<\infty \text { implies that } \mu=\tau \times_{T} \nu \text { has a solution } \nu \text {. }
$$

This sufficient condition is more general than $\int_{Y} \tau d \mu<\infty$ since $\rho \leq \tau$. (However, it is not necessary, as Example 2.3 shows, where $Y=X$ and hence $\rho=\tau$.)

\section{A generalized Abramov formula for the entropy}

For $(X, \mathcal{A}, \mu) \sigma$-finite and $Y \in \mathcal{A}^{+}(\mu)$ with $\mu(Y)<\infty$, we let $\mu_{Y}:=\mu(Y)^{-1}$. $\left.\mu\right|_{\mathcal{A} \cap Y}$, the normalized restriction of $\mu$ to $Y$. Abramov's classical entropy formula for first return maps states that whenever $(X, \mathcal{A}, \mu, T), \mu(X)<\infty$, is an ergodic measure-preserving system and $Y \in \mathcal{A}^{+}(\mu)$, then

$$
h\left(T_{Y}, \mu_{Y}\right)=\frac{\mu(X)}{\mu(Y)} \cdot h\left(T, \mu_{X}\right)=\int_{Y} \varphi_{Y} d \mu_{Y} \cdot h\left(T, \mu_{X}\right) .
$$

We are going to show that an analogous relation holds for general induced transformations $T^{\tau}$, even in the case of infinite measures. In order to state the result in full generality, we recall Krengel's notion of entropy for conservative systems (cf. $[\mathrm{Kr}])$ : For any c.e.m.p.t. $T$ on $(X, \mathcal{A}, \mu)$, 5.1 shows that

$$
h(T, \mu):=\mu(Z) \cdot h\left(T_{Z}, \mu_{Z}\right)
$$

where $Z \in \mathcal{A}^{+}(\mu), \mu(Z)<\infty$, does not depend on the choice of $Z$ and therefore defines the entropy of $T$ w.r.t. $\mu$ unambiguously. (Combined with other characteristics like minimal wandering rates or asymptotic type, this yields rather strong isomorphism invariants if $\mu(X)=\infty$; cf. [A1] or [Th].) Note that using this formalism, Abramov's classical result (5.1) simply becomes

$$
h\left(T_{Y},\left.\mu\right|_{\mathcal{A} \cap Y}\right)=h(T, \mu),
$$

if we abstain from normalizing the measures. (If $\mu=\tau \times_{T} \nu$, then $\mu$ and $\nu$ have infinite or different total mass unless $\tau \equiv 1$.)

Theorem 5.1 (Generalized Abramov formula). Let $T$ be a c.e.m.p.t. on the $\sigma$ finite measure space $(X, \mathcal{A}, \mu)$, and let $\tau$ be an inducing time $(\bmod \mu)$ for $T$ on $Y \in \mathcal{A}^{+}(\mu)$. Assume that $T^{\tau}$ has an invariant measure $\nu \ll \mu$ satisfying $\mu=\tau \times_{T} \nu$. Then the respective entropies coincide:

$$
h\left(T^{\tau}, \nu\right)=h(T, \mu)
$$

If $\mu$ and $\nu$ are finite and we normalize them, this amounts to

$$
h\left(T^{\tau}, \nu_{Y}\right)=\frac{\mu(X)}{\nu(Y)} \cdot h\left(T, \mu_{X}\right)=\int_{Y} \tau d \nu_{Y} \cdot h\left(T, \mu_{X}\right) .
$$


Proof. (i) Consider finite measures $\mu, \nu$ first. We are going to use the representation of $T^{\tau}$ in terms of the $\tau$-sepaparting extension developed in Section 2. By statement (3.4) in the proof of Theorem 1.1, $\mu$ lifts to an a.c.i.m. $\widehat{\nu}$ for the nonsingular extension $(\widehat{X}, \widehat{\mathcal{A}}, \widehat{\mu}, \widehat{T})$, and $\nu_{Y}=\left(\widehat{\nu}_{\widehat{Y}_{0}}\right) \circ \pi^{-1}$. By the classical Abramov formula for the induced map $\widehat{T}_{\widehat{Y}_{0}}$ of the tower system we have

$$
h\left(\widehat{T}_{\widehat{Y}_{0}}, \widehat{\nu}_{\widehat{Y}_{0}}\right)=\int_{\widehat{Y}_{0}} \widehat{\varphi}_{\widehat{Y}_{0}} d \widehat{\nu}_{\widehat{Y}_{0}} \cdot h\left(\widehat{T}, \widehat{\nu}_{\widehat{X}}\right) .
$$

Due to the natural isomorphism of the m.p.t.s. $\left(\widehat{Y}_{0}, \widehat{\mathcal{A}} \cap \widehat{Y}_{0}, \widehat{\nu}_{\widehat{Y}_{0}}, \widehat{T}_{\widehat{Y}_{0}}\right)$ and $(Y, \mathcal{A} \cap$ $\left.Y, \nu_{Y}, T^{\tau}\right)$, it is clear that $h\left(\widehat{T}_{\widehat{Y}_{0}}, \widehat{\nu}_{\widehat{Y}_{0}}\right)=h\left(T^{\tau}, \nu_{Y}\right)$, and since $\left.\widehat{\varphi}_{\widehat{Y}_{0}}\right|_{\widehat{Y}_{0}}=\left.\tau \circ \pi\right|_{\widehat{Y}_{0}}$, we see that $\int_{\widehat{Y}_{0}} \widehat{\varphi}_{\widehat{Y}_{0}} d \widehat{\nu}_{\widehat{Y}_{0}}=\int_{Y} \tau d \nu_{Y}$. Hence,

$$
h\left(T^{\tau}, \nu_{Y}\right)=\int_{Y} \tau d \nu_{Y} \cdot h\left(\widehat{T}, \widehat{\nu}_{\widehat{X}}\right) .
$$

It remains to check that $h\left(\widehat{T}, \widehat{\nu}_{\widehat{X}}\right)=h\left(T, \mu_{X}\right)$. This, however, is automatic for any extension with countable fibres; cf. [Bu], Proposition 2.8.

(ii) Extension to possibly infinite measures. Take any $Z \in \mathcal{A}^{+}(\nu), \nu(Z)<\infty$, so that $0<\mu(Z)<\infty$ as well, and (5.2) applies. Then

$$
h\left(T^{\tau}, \nu\right)=\nu(Z) \cdot h\left(\left(T^{\tau}\right)_{Z}, \nu_{Z}\right)=\nu(Z) \cdot h\left(\left(T_{Z}\right)^{\vartheta}, \nu_{Z}\right),
$$

where $\vartheta$ is such that $\left(T^{\tau}\right)_{Z}=\left(T_{Z}\right)^{\vartheta}$, and $\left.\mu\right|_{\mathcal{A} \cap Z}=\vartheta \times\left._{T_{Z}} \nu\right|_{\mathcal{A} \cap Z}$ as in Proposition 4.1. By the finite measure version of our theorem and Proposition 1.1] $h\left(\left(T_{Z}\right)^{\vartheta}, \nu_{Z}\right)=(\mu(Z) / \nu(Z)) \cdot h\left(T_{Z}, \mu_{Z}\right)$, and (5.3) follows.

\section{ACKNOWLEDGMENTS}

I am indebted to J. Buzzi and O. Sarig for asking for the entropy formula and suggesting the quick proof for finite measures. I also thank the Mathematics Department at Warwick University, where much of this work was done, for their generous hospitalty.

\section{REFERENCES}

[A0] J.Aaronson: An introduction to infinite ergodic theory. American Mathematical Society, Mathematical Surveys and Monographs, Vol. 50, 1997. MR1450400 (99d:28025)

[A1] J.Aaronson: Rational ergodicity and a metric invariant for Markov shifts. Israel J. Math. 27 (1977), 93-123. MR0584018 (58:28424)

[Ab] L.M.Abramov: Entropy of a derived automorphism. Amer. Math. Soc. Transl. Ser. II, 49 (1960), 162-176.

[Br] H.Bruin: Induced maps, Markov extensions and invariant measures in one-dimensional dynamics. Commun. Math. Phys. 168 (1995), 571-580. MR.1328254 (96m:58134)

[Bu] J.Buzzi: Markov extensions for multi-dimensional dynamical systems. Israel J. Math. 112 (1999), 357-380. MR1714974 (2000m:37008)

[DS] N.Dunford, J.T.Schwartz: Linear operators I. Wiley 1957.

[He] G.Helmberg: Über konservative Transformationen. Math. Annalen 165 (1966), 44-61. MR0197679 (33:5842)

[Ka] S.Kakutani: Induced measure preserving transformations. Proc. Imp. Acad. Sci. Tokyo 19 (1943), 635-641. MR0014222 (7:255f)

[Ke] G.Keller: Lifting measures to Markov extensions. Mh. Math. 108 (1989), 183-200. MR:1026617 (91b:28011)

[Kr] U.Krengel: Entropy of conservative transformations. Z. Wahrscheinlichkeitstheorie verw. Geb. 7 (1967), 161-181. MR0218522 (36:1608)

[PS] Y.Pesin, S.Senti: Equilibrium states for unimodal maps. Preprint 2003. 
[Sc] F.Schweiger: Ergodic theory of fibered systems and metric number theory. Clarendon Press, Oxford 1995. MR1419320 (97h:11083)

[Th] M.Thaler: Transformations on $[0,1]$ with infinite invariant measures. Israel J. Math. 46 (1983), 67-96. MF 0727023 (85g:28020)

Department of Mathematics, Imperial College London, 180 Queen's Gate, London SW7 2AZ, United KINGDOM

E-mail address: r.zweimueller@imperial.ac.uk 